\title{
Corrections to linear mixing in binary ionic mixtures and plasma screening at zero separation
}

\author{
Andrey I. Chugunov ${ }^{1, *}$ and Hugh E. DeWitt ${ }^{2}$ \\ ${ }^{1}$ Ioffe Institute, Politekhnicheskaya 26, 194021 St.Petersburg, Russia \\ ${ }^{2}$ Lawrence Livermore National Laboratory, Livermore, California 94550, USA
}

Received XXXX, revised XXXX, accepted XXXX

Published online XXXX

Key words Screening, nonideal plasma, mixing rule

PACS 52.27.Gr, 26.20.Np, 97.10.Cv, 97.60.Jd

Using the results of extensive Monte Carlo simulations we discuss corrections to the linear mixing rule in strongly coupled binary ionic mixtures. We analyze the plasma screening function at zero separation, $H_{j k}(0)$, for two ions (of types $j=1,2$ and $k=1,2$ ) in a strongly coupled binary mixture. The function $H_{j k}(0)$ is estimated by two methods: (1) from the difference of Helmholtz Coulomb free energies at large and zero separations; (2) by fitting the Widom expansion of $H_{j k}(x)$ in powers of interionic distance $x$ to Monte Carlo data on the radial pair distribution function $g_{j k}(x)$. These methods are shown to be in good agreement. For illustration, we analyze the plasma screening enhancement of nuclear burning rates in dense stellar matter.

Copyright line will be provided by the publisher

\section{Introduction}

More than 30 years ago [1] the linear mixing rule for multicomponent strongly coupled mixtures was shown to be highly accurate. However, only recent studies $[2,3]$ have achieved enough accuracy to describe the corrections to the linear mixing rule for a wide range of plasma parameters; previous attempts, e.g. [4,5], were restricted at least by a limited number of data points. We discuss the corrections to the linear mixing rule in application to the plasma screening of nuclear reactions in strongly coupled mixtures. Following Ref. [6] we apply two approaches to calculate the screening enhancement: one is based on the thermodynamic relations and the other on fitting the mean-field potentials. The main advance of the present work is in using a much wider set of numerical data and most precise thermodynamic results.

\section{Plasma screening enhancement of nuclear reaction rates}

Let us study a multicomponent mixture of ions $j=1,2, \ldots$ with atomic mass numbers $A_{j}$ and charge numbers $Z_{j}$. The ions are supposed to be fully ionized. Their total number density is the sum of partial densities, $n_{\mathrm{i}}=$ $\sum_{j} n_{j}$. It is useful to introduce the fractional number $x_{j}=n_{j} / n_{\mathrm{i}}$ of ions $j$. Let us also define the average charge number $\langle Z\rangle=\sum_{j} x_{j} Z_{j}$ and mass number $\langle A\rangle=\sum_{j} x_{j} A_{j}$ of the ions. The charge neutrality implies that the electron number density is $n_{\mathrm{e}}=\langle Z\rangle n_{\mathrm{i}}$. The electron plasma screening is typically weak and will be neglected.

Thermonuclear reactions in stars take place after the atomic nuclei collide and penetrate through the Coulomb barrier. For a not too cold and dense stellar matter the tunneling length $r_{\mathrm{t}}$ is much smaller than interionic distances (for recent result of nuclear fusion with large tunneling distances see $[7,8]$ ). The interaction of the reacting ions with neighboring plasma particles creates a potential well which enlarges the number of close encounters and enhances the reaction rate. Before the tunneling event the reactants $j$ and $k$ behave as classical particles. Their correlations can be described by the classical radial pair distribution function $g_{j k}(r)$. It can be calculated by the classical Monte Carlo technique and written as $g_{j k}(r)=\exp \left[-\Gamma_{j k} a_{j k} / r+H_{j k}(r) / T\right]$, where $\Gamma_{j k}=Z_{j} Z_{k} e^{2} /\left(a_{j k} T\right)$ is the correponding Coulomb coupling parameter, and $T$ is the temperature. The ion

\footnotetext{
* Corresponding author E-mail: andr.astro@mail.ioffe.ru, Phone: +7812 292 7180, Fax: +7 8122971017
} 
sphere radius $a_{j k}$ can be defined as [9] $a_{j k}=\left(a_{j}+a_{k}\right) / 2$ and $a_{j}=Z_{j}^{1 / 3} a_{\mathrm{e}}$, where $a_{\mathrm{e}}=\left(4 \pi n_{\mathrm{e}} / 3\right)^{-1 / 3}$. The function $H_{j k}(r)$ is the mean-field plasma potential. The plasma enhancement factor is then given by $F_{j k}\left(r_{\mathrm{t}}\right)=g_{j k}\left(r_{\mathrm{t}}\right) / g_{j k}^{\mathrm{id}}\left(r_{\mathrm{t}}\right)=\exp \left[H_{j k}\left(r_{\mathrm{t}}\right) / T\right] \approx \exp \left[H_{j k}(0) / T\right]$. Here, $g_{j k}^{\mathrm{id}}\left(r_{\mathrm{t}}\right)=\exp \left(-\Gamma_{j k} a_{j k} / r_{\mathrm{t}}\right)$ is the pair distribution function in the absence of screening. In the last equality we neglect variations of $H_{j k}(r)$ over scales $\sim r_{\mathrm{t}}$ which are much lower than scales $\sim a_{j k}$ of $H_{j k}(r)$.

Widom expansion. The enhancement factor of nuclear reaction rates can be determined in following way: one can calculate $g_{j k}(r)$ by classical Monte Carlo, extract $H_{j k}(r)$ and extrapolate the results to $H_{j k}(0)$. The extrapolation is delicate [10] because of poor Monte Carlo statistics at small separations. We expect that the expansion of $H_{j k}(r)$ contains only even powers of $r / a_{j k}$ (the Widom expansion, [11]); its quadratic term is known [12]:

$$
H_{j k}(r)=H_{0}-\frac{Z_{j} Z_{k} e^{2}}{2 a_{j k}^{\text {comp }}}\left(\frac{r}{a_{j k}^{\text {comp }}}\right)^{2}+H_{4}\left(\frac{r}{a_{j k}}\right)^{4}-H_{6}\left(\frac{r}{a_{j k}}\right)^{6}+\ldots
$$

Here, $H_{0}=H_{j k}(0)$ and $a_{j k}^{\text {comp }}=\left(Z_{j}+Z_{k}\right)^{1 / 3} a_{\mathrm{e}}$ is the ion-sphere radius of the compound nuclei. Let us also introduce the dimensionless parameter $h_{j k}^{0}=H_{j k}(0) / T$. We have performed a large number of Monte Carlo simulations of mean field potentials in binary ionic mixtures. For each simulation, we fit $H_{j k}(r)$ by Eq. (1) taking $H_{0}, H_{4}$ and $H_{6}$ as free parameters. To estimate error bars we have varied $H_{0}$ and made additional fits with two free parameters, $H_{4}$ and $H_{6}$. Fig. 1 1 shows the normalized enhancement parameter $h_{j k}^{0} / \Gamma_{j k}$ (dots with error bars) calculated in this way.

Thermodynamic enhancement factors. The second approach to calculate the enhancement factors comes from thermodynamics. One can estimate $H_{j k}(0)$ as a difference of the Helmgoltz Coulomb free energies $F$ of the system before and after the reaction event (e.g., [13]):

$$
h_{j k}^{0}=\left[F\left(\ldots, N_{j}, N_{k}, N_{j k}^{\mathrm{comp}}, \ldots\right)-F\left(\ldots, N_{j}-1, N_{k}-1, N_{j k}^{\mathrm{comp}}+1, \ldots\right)\right] / T,
$$

where $N_{j}, N_{k}, N_{j k}^{\text {comp }}$ are the numbers of the reacting nuclei and the compound nuclei $\left(Z_{j}+Z_{k}, A_{j}+A_{k}\right)$.

Usually (see, e.g. [8]) one assumes the linear mixing model and presents the free energy of the Coulomb mixture $F$ as $F^{\text {lin }}\left(\left\{N_{j}\right\}\right)=T \sum_{j} N_{j} f_{0}\left(\Gamma_{j j}\right)$, where $f_{0}(\Gamma)$ is the Coulomb free energy (normalized to temperature $T)$ per one nucleus in one component plasma. We use the well known approximation of $f_{0}(\Gamma)$ suggested by Potekhin \& Chabrier [14]. In linear mixing model Eq. (2) can be written in the convenient form:

$$
h_{j k}^{\operatorname{lin}}=f_{0}\left(\Gamma_{j j}\right)+f_{0}\left(\Gamma_{k k}\right)-f_{0}\left(\Gamma_{j k}^{\mathrm{comp}}\right),
$$

where $\Gamma_{j k}^{\text {comp }}=\left(Z_{j}+Z_{k}\right)^{5 / 3} \Gamma_{\mathrm{e}}$ is the Coulomb coupling parameter for the compound nucleus. The values of $h_{j k}^{\operatorname{lin}}$ are shown by the solid line in Fig. 1

Our aim is to check the accuracy of the linear mixing and analyze deviations from this model. To do this we apply the best available results for the thermodynamics of multicomponent mixtures [2,3], which are valid for any value of the coupling parameter. The values of the corresponding enhancement parameter $h_{j k}^{0} / \Gamma_{j k}$ are shown by the long-dash line in Fig. 1

\section{Comparison of different approaches}

In Fig. 1 we compare the plasma screening function at zero separation calculated by different methods. Each of six panels demonstrates the histogram of normalized screening functions $h_{j k}^{0} / \Gamma_{j k}$ versus simulation number. Three left panels show simulations with numbers from 1 to 50, and three right panels show simulations from 51 to 100 . For each 3-panel block, the lower panel presents $h_{11}^{0} / \Gamma_{11}$, the middle panel shows $h_{12}^{0} / \Gamma_{12}$, and the upper panel gives $h_{22}^{0} / \Gamma_{22}$. The parameters of simulations $\left(\Gamma_{11}, Z_{2} / Z_{1}, x_{1}\right)$ are also shown on each block by vertically aligned numbers: $\Gamma_{11}$ on the lower panel, $Z_{2} / Z_{1}$ on the middle and $x_{1}$ on the upper panel. For example, the simulation number 1 is done for $\Gamma_{11} \approx 0.33, Z_{2} / Z_{1}=2$, and $x_{1}=0.7$. 

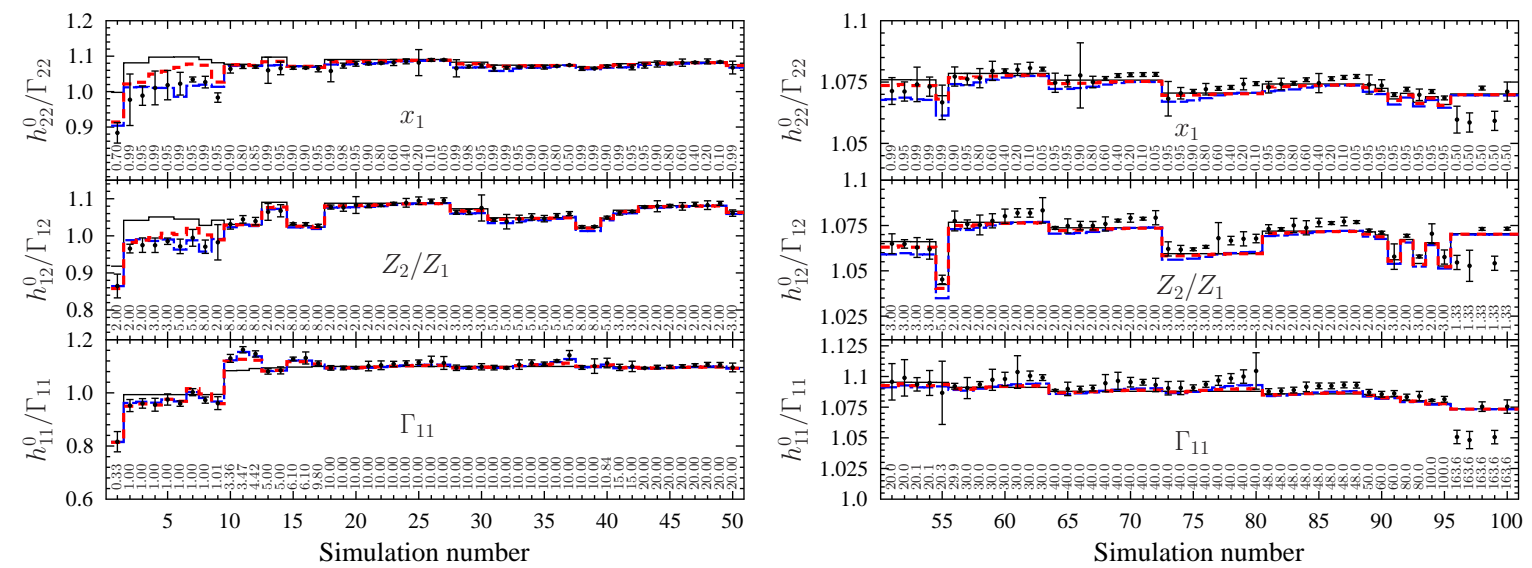

Fig. 1 (Color online) Histogram of the enhancement factors extracted from: 1) Widom fitting (dots with error bars); 2) linear mixing [Eq. (3); solid line]; 3) thermodynamics (long-dash lines); 4) our approximation [Eq. (4); short-dash lines].

Each panel contains a set of dots with error bars, which represent the values of $h_{j k}^{0} / \Gamma_{j k}$ calculated by fitting $H_{j k}(r)$ with the aid of (1). Each panel contains 3 lines: the solid line shows the results of the linear mixing model, Eq. (3); the long-dash line is calculated with the best available thermodynamics of the multicomponent plasma (Eq. (2) with the free energy taken from [3]); the short-dash line is our approximation (4). Note, that the normalized enhancement parameter is approximately constant at large $\Gamma_{j k}$. This property is well known [15]. The linear mixing is a highly accurate as long as $\Gamma_{j k} \gtrsim 10$. For lower $\Gamma_{j k}$ the relative corrections can be much larger and well described by both dashed-lines (the accurate thermodynamics and approximation). The most noticeable difference between dots and the short-dashed lines takes place for $h_{22} / \Gamma_{22}$ in simulations 6, 7, 8, and 9 that are done for low fractions of highly charged ions and large ratio $Z_{2} / Z_{1} \geq 5$. Such a difference is unimportant for applications - it translates into the correction to the reaction rate within a factor of two.

Also, there are three large $\Gamma$ simulations $(96,97$, and 99), where dots are divergent. They started with lattice configurations of ions. Thus the corrections to the linear mixing in crystalline phase are larger (as noted in [5]).

\section{Approximation of enhancement factors and conclusions}

We suggest to use the following approximation for the enhancement factor for all $\Gamma$ and mixture composition

$$
h_{j k}^{0}=h_{j k}^{\operatorname{lin}} /\left[1+C_{j k}\left(1-C_{j k}\right)\left(h_{j k}^{\operatorname{lin}} / h_{j k}^{\mathrm{DH}}\right)^{2}\right]
$$

Here, $h_{j k}^{\text {lin }}$ is given by (3), $h_{j k}^{\mathrm{DH}}=3^{1 / 2} Z_{j} Z_{k}\left\langle Z^{2}\right\rangle^{1 / 2} \Gamma_{\mathrm{e}}^{3 / 2} /\langle Z\rangle^{1 / 2}$ is the well known Debye-Hückel enhancement parameter, and $C_{j k}=3 Z_{j} Z_{k}\left\langle Z^{2}\right\rangle^{1 / 2}\langle Z\rangle^{-1 / 2} /\left[\left(Z_{j}+Z_{k}\right)^{5 / 2}-Z_{j}^{5 / 2}-Z_{k}^{5 / 2}\right]$. Eq. (4) reproduces the Debye-Hückel asymptote at low $\Gamma$ and the linear mixing at strong coupling.

In Fig. 2 we show the dependence of the approximated enhancement factors $h_{j k}^{0} / \Gamma_{11}^{3 / 2}$ on $\Gamma_{11}$. The figure contains three panels; each for a specific binary ionic mixture. Each panel shows three groups of four lines. They are (from top to bottom) $h_{22}^{0} / \Gamma_{1}^{3 / 2}, h_{12}^{0} / \Gamma_{1}^{3 / 2}$ and $h_{11}^{0} / \Gamma_{1}^{3 / 2}$. Two of any four lines (solid and thick dashed lines) are almost the same in the majority of cases. This couple represents the approximation (4) and the thermodynamic enhancement factor (2), respectively. The dotted horizontal lines refer to the Debye-Hückel model and the dash-dot lines are the linear mixing results. One can see that our approximation is in a good agreement with thermodynamic results for most of cases, especially in panel (a) (for all mixtures with not too large $Z_{2} / Z_{1}$ ). If $Z_{2} / Z_{1}$ becomes too large [panel (c)], the thermodynamic model of $h_{11}^{0} / \Gamma_{1}$, calculated in accordance with [3], has a specific feature $\left(h_{11} / \Gamma_{11}^{3 / 2}\right.$ increases at $\left.\Gamma_{11} \sim 10^{-2}\right)$, while our approximation has not. We expect that this feature is not real, but results from not too accurate extractions of the enhancement factors from thermodynamic data. The free energy is almost fully determined by larger charges $Z_{2}$ which also dominate by number (99\%) in 

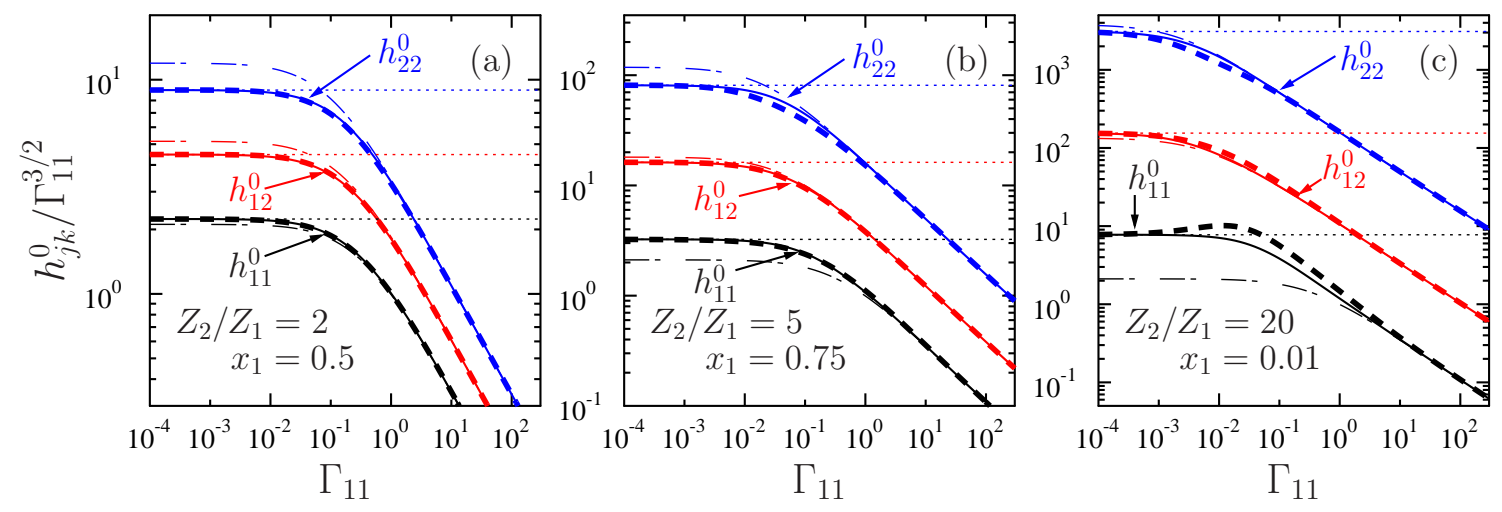

Fig. 2 (Color online) Enhancement factors $h_{j k}^{0} / \Gamma_{11}^{3 / 2}$ vs $\Gamma_{11}$ for three binary ionic mixtures.

panel (c). Using Eq. 2 to get $h_{11}^{0}$, one should differentiate the free energy with respect to $N_{1}$, which provides vanishing contribution to the free energy. Hence this procedure is very delicate and can strongly amplify the errors of original thermodynamic approximation. We expect that our approximation can be more accurate than the original thermodynamic result. Another, less probable option is that we still have not enough data to prove the presence of the feature of $h_{11}^{0} / \Gamma_{11}^{3 / 2}$.

To conclude, we have calculated the enhancement factors of nuclear reactions in binary ionic mixtures by two methods and showed good agreement of the results. We have proposed a simple approximation of the enhancement factors valid for any Coulomb coupling. This approximation is almost the same as thermodynamic ones for not too specific mixtures. It does not confirm some questionable features of the enhancement factors for mixtures with large $Z_{2} / Z_{1}$ and small $x_{1}$.

Acknowledgements We are grateful to D.G. Yakovlev and A.Y. Potekhin for useful remarks. Work of AIC was partly supported by the Russian Foundation for Basic Research (grant 08-02-00837), and by the State Program "Leading Scientific Schools of Russian Federation" (grant NSh 2600.2008.2). Work of HED was performed under the auspices of the US Department of Energy by the Lawrence Livermore National Laboratory under contract number W-7405-ENG-48.

\section{References}

[1] J. P. Hansen and P. Vieillefosse, Phys. Rev. Lett. 37, 391 (1976).

[2] A. Y. Potekhin, G. Chabrier, F. J. Rogers, Phys. Rev. E 79, 016411 (2009).

[3] A. Y. Potekhin, G. Chabrier, A. I. Chugunov, H. E. DeWitt, F. J. Rogers, Phys. Rev. E, submitted

[4] H. DeWitt, W. Slattery, G. Chabrier, Physica B 228, 21 (1996).

[5] H. DeWitt, W. Slattery, Contrib. Plasma Phys. 43, 279 (2003)

[6] H. DeWitt, W. Slattery, Contrib. Plasma Phys. 39, 97 (1999)

[7] A. I. Chugunov, H. E. DeWitt, D.G. Yakovlev, Phys. Rev. D 76, 025028 (2007).

[8] A. I. Chugunov, H. E. DeWitt, Phys. Rev. C, 80, 014611 (2009)

[9] N. Itoh, F. Kuwashima, and H. Munakata, Astrophys. J. 362, 620 (1990).

[10] Y. Rosenfeld, Phys. Rev. E 53, 2000 (1996).

[11] B. Widom, J. Chem. Phys. 39, 2808 (1963).

[12] S. Ogata, H. Iyetomi, and S. Ichimaru, Astrophys. J. 372, 259 (1991).

[13] D. G. Yakovlev and D. A. Shalybkov, Soviet Sci. Rev. Sec. E 7, 313 (1989).

[14] A. Y. Potekhin and G. Chabrier, Phys. Rev. E 62, 8554 (2000).

[15] E. E. Salpeter, Aust . J. Phys. 7, 373 (1954). 